How to reference this article

Łukaszewicz, J. (2021). La Padova di August Fryderyk Moszyński. Italica Wratislaviensia, 12(1), 123-140.

DOI: http://dx.doi.org/10.15804/IW.2021.12.1.07

Justyna Łukaszewicz

Uniwersytet Wrocławski, Polonia

justyna.lukaszewicz@uwr.edu.pl

ORCID: 0000-0003-2140-3610

\title{
LA PADOVA \\ DI AUGUST FRYDERYK MOSZYŃSKI
}

\section{THE PADUA OF AUGUST FRYDERYK MOSZYŃSKI}

\begin{abstract}
This article is dedicated to the image of Padua in the diary of August Fryderyk Moszyński (1731-1786), a close collaborator of King Stanislas August Poniatowski, architect, director of royal buildings, and promoter of the Warsaw theatre. The diary, which covers a journey to France and Italy (1784-1786), has been partially published in the original French version $(1930 ; 2010)$ and Polish translation (1970). The diary is remarkable for Polish $18^{\text {th }}$-century odeporic literature due to its critical approach, quasi-scientific character, and interest in contemporary developments. In this article, the author establishes the dates of Moszyński's stay in Padua and then analyses elements such as the descriptions, impressions, and assessments of urban environments; buildings, pictures, people, and events; as well as cultural and scientific life. The portrait of Padua drawn by Moszyński is compared with that which emerges from other travelogues and texts presented to the Polish public at the time, in particular with the description of Italy that appeared in the Warsaw journal Pamiętnik HistorycznoPolityczny in 1787. The analysis of the travelogue passages relating to the two stays in Padua confirms the versatility of the traveller. The description of Padua by Moszyński is multifaceted, often ironic and personal. He wrote as an expert in architecture and urban spaces; a connoisseur of theatre; a builder of scientific instruments; an admirer of Renaissance artists; a lover of truth in art; and a sharp and critical observer. The last part of the article is dedicated to the circumstances of Moszyński's death, which, contrary to what may be suggested by the diary, did not occur in Padua but in Venice (on 3 July 1786, at the famous Leon Bianco inn).
\end{abstract}

Keywords: August Fryderyk Moszyński, Padua, travelogue, Italy, literary image 


\section{L'ODEPORICO E IL SUO AUTORE}

I1 conte August Fryderyk Moszyński, nipote naturale di Augusto II, nacque a Dresda nel 1731 (Wierzbicka-Michalska, 1977, p. 108) e cominciò una brillante carriera alla corte sassone di Augusto III per diventare poi amico e stretto collaboratore del suo successore al trono della Polonia, Stanislao Augusto Poniatowski (1764-1794). Direttore di edifici reali, sovrintendente del teatro di Varsavia, collezionista e curatore di collezioni reali, massone di spicco, alchemico e denunciatore di Cagliostro, Moszyński era anche un noto spendaccione e amante del fascino femminile. Negli anni 1784-1786, viaggiò in Francia e in Italia a spese del re, scrivendo un diario in francese.

Il manoscritto di questo odeporico è conservato nella Biblioteca dei Principi Czartoryski (Biblioteka Książąt Czartoryskich), filiale del Museo Nazionale di Cracovia ${ }^{1}$. Un brano relativo alla Provenza è stato pubblicato in Francia nel 1930. Più recentemente Guillaume Calafat ha curato la pubblicazione del secondo quaderno, dedicato all'itinerario Vienne (nel Delfinato) - Avignon - Nîmes - Montpellier - Arles - Aix Marseille (2010). L'edizione più completa del diario comprende una versione polacca con tagli operati dalla traduttrice, Bożena ZboińskaDaszyńska (Moszyński, 1970).

Moszyński era un uomo di ampie conoscenze, tra l'altro in architettura, studiata sotto la guida di Gaetano Chiaveri (architetto alla corte di Augusto III) e durante il primo, giovanile viaggio in Italia, poi praticata attraverso progetti di chiese e teatri (Mańkowski, 1930). Il suo diario, "una delle opere più critiche e più scientifiche della letteratura di viaggio polacca" dell'epoca (Wyrzykowska, 2012, p. 20), costituisce una buona

1 Il diario era composto di sette quaderni, di cui tre non ci sono pervenuti. Quella che ci interessa in questa sede è l'ultima parte del diario, composta di 189 pagine $(27,5 \times 20 \mathrm{~cm})$ del quaderno n. VII, intitolata "Retour de Naples à Rome, la continuation des observations sur cette dernière ville, ainsi que le voyage de Rome à Florence et de Florence et Padoue à Venise", collocazione: 1535 III Rkps, versione elettronica: https://cyfrowe.mnk.pl/dlibra/publication/24395\%20(Biblioteka\%20Ksi???t\%20 Czartoryskich). 
testimonianza della vastità di interessi e la parte dedicata a Padova ne fornisce un buon esempio.

"Moszyński era un tipo di dignitario di corte sassone-polacco, la cui patria e sede poteva essere sia la Sassonia sia la Polonia" (Mańkowski, 1930, p. 172), cittadino della Repubblica delle Lettere e uomo dotato di grande capacità di adattamento. Durante il suo soggiorno a Venezia, osserva: "Viaggiando si diventa cosmopoliti; il luogo in cui ci si trova diventa per un po' una patria d'adozione. È giusto e prudente amare questa patria" (Moszyński, 1970, p. 584; Ms., p. 96).

Tra i motivi ricorrenti nel diario di Moszyński, ci sono la sua scarsa vista (menzionata ben quattro volte nei brani riguardanti Padova) e il viaggio in Italia compiuto a meno di vent'anni. L'autore nota ad esempio: “Ho trovato Venezia quasi com'era nel 1747” (ibid., p. 565; Ms., p. 61)2. A proposito del metodo comparativo, da lui preferito (Kostkiewiczowa, 1988, p. 206), il viaggiatore scrive: "Mi piacciono i paragoni. Spesso mi chiariscono cose inizialmente incomprensibili" (ibid., p. 252). La comparazione viene iscritta anche nella definizione dello scopo assegnato all'odeporico: "uno dei compiti di questo diario di viaggio è quello di spiegare lo stato attuale delle belle arti e le ragioni del loro progresso o declino" (ibid., p. 258). Un paragone più generalizzato, unito al disinganno di chi ha visto e vissuto tante cose, si fa sentire nell'espressione nihil novi sub sole, che appare due volte nella relazione del primo soggiorno padovano (ibid., p. 563; Ms., pp. 58-59).

Moszyński, "esperto della letteratura di viaggio del suo tempo" (Wyrzykowska, 2012, p. 20), ha utilizzato, tra le diverse altre fonti, la famosa relazione di viaggio pubblicata dall'astronomo francese Jérome Lalande (1769). Il viaggiatore polacco l'ha spesso citata nel suo diario, senza copiare le informazioni in essa contenute (Wrześniak, 2010, p. 17). A Firenze ha scritto: "Mi limiterò, come in altre città italiane,

2 Tutte le traduzioni in italiano, dei brani di testi polacchi o francesi che cito nell'articolo, sono mie. Per quanto riguarda il diario, prendo come punto di partenza la versione polacca (Moszyński, 1970), verificando - nel caso dei frammenti relativi a Padova o Venezia - se i passaggi riportati corrispondono al manoscritto di Moszyński. 
a parlare di ciò che mi tocca" (Moszyński, 1970, p. 162). Questo vale anche per le sue annotazioni su Padova.

\section{L'OGGETTO DELLO STUDIO E L'APPROCCIO METODOLOGICO}

Tra i numerosi studiosi che si occuparono delle varie manifestazioni dei rapporti culturali, che unirono nel passato Polonia e Italia, tra cui i viaggi, mi limiterò a menzionare Henryk Barycz, autore del libro Spojrzenia w przeszłość polsko-włoska (1965). Negli ultimi anni fioriscono edizioni e studi di diari di viaggiatori polacchi in Italia, in particolare a cura di Bogdan Rok, Filip Wolański, Małgorzata Ewa Kowalczyk, Adam Kucharski. Il presente articolo si colloca in questo campo di ricerca.

In base a una serie di informazioni indirette fornite dallo stesso diario di Moszyński, si fanno delle ipotesi relative alle date dei soggiorni del conte a Padova. In seguito, vengono analizzati elementi e aspetti dell'immagine letteraria di questa città, quali descrizioni, impressioni e valutazioni relative ad ambienti urbani, edifici, quadri, persone ed eventi, nonché la vita culturale e scientifica. Il ritratto di Padova disegnato da Moszyński è messo a confronto con quanto osservato da altri viaggiatori polacchi della stessa epoca, ma anche con quello che emerge da testi su argomenti simili presentati al pubblico polacco a quel tempo, in particolare dalla descrizione dell'Italia apparsa a puntate sulla rivista varsaviana Pamiętnik Historyczno-Polityczny nel 1787. Nel corso dell'analisi, faccio riferimento allo stato delle ricerche sull'odeporico relative ad aspetti trasversali (Wyrzykowska, 2012) e punti particolari (Lenart, 2005; Lenart 2005a).

\section{LE DATE DEL SOGGIORNO DI MOSZYŃSKI A VENEZIA E A PADOVA}

L'ultima parte del diario, dedicata al viaggio dal sud al nord d'Italia e al soggiorno nel Veneto $^{3}$, si apre con l'informazione sulla partenza, il 4

${ }^{3}$ Nell'edizione del 1970 occupa 50 pagine (551-561), nel manoscritto (Ms.) $79(51-129)$. 
maggio 1786, da Napoli, dove Moszyński trascorse nove mesi. Il conte giunse a Padova attraverso Terracina, Velletri, Albano, Roma, Siena, Firenze, Bologna e Ferrara ${ }^{4}$. Da Padova a Venezia, destinazione agognata nel corso del viaggio cominciato quasi due anni prima a Varsavia (Moszyński, 1970, pp. 563-564; Ms., p. 59), Moszyński arrivò - dopo un tragitto di otto ore sul Brenta, eseguito con una chiatta trainata da cavalli - il giorno dell'Ascensione (ibid., p. 564-565; Ms., pp. 59-62), che nel 1786 cadeva il 25 maggio (cf. Wiki/1786). Quindi deve essere stato a Padova il 24 maggio 1786.

A Venezia Moszyński trascorse almeno tre settimane (cf. Moszyński, 1970, p. 587; Ms., p. 104) e assistette alla processione del Corpus Domini (ibid., p. 579; Ms., p. 89) che cadde il 15 giugno del 1786. Dopo quel giorno il conte tornò a Padova per assistere alla corsa dei barberi nel Prato della Valle. Il suo resoconto di questo evento completa la principale nota su Padova e chiude sia il quaderno che l'intero diario. Nel manoscritto, la prima nota su Padova occupa più di sette pagine (pp. 51-59), la seconda quasi due (pp. 127-129).

\section{LA DECADENZA DI PADOVA}

La nota relativa al primo soggiorno a Padova si apre con una osservazione indicativa dell'atteggiamento piuttosto negativo del viaggiatore: "È una città abbastanza grande, ma poco popolata da quando la sua università e la fama di Sant'Antonio hanno perso il loro splendore" (ibid., p. 559; Ms., p. 51). L'opposizione tra le grandi dimensioni della città e la sua scarsa popolazione ricorre così spesso nei testi dei viaggiatori polacchi dell'epoca che sembra un luogo comune: lo osservano Kazimierz Symforian Arakiełowicz nel 1723 (Kowalczyk, 2005, p. 220), Ignacy Potocki nel 1765 (Rok \& Wolański, 2016, p. 119), Teofila Konstancja Morawska nel 1774 (Morawska, 2002, p. 133), Franciszek Ksawery

4 Oltre al giorno della partenza da Napoli, non ci sono altre date in questo brano del diario, Moszyński scrive però che passò a Roma una settimana, a Firenze - quattro giorni, a Bologna - qualche ora. 
Bohusz nel 1781 (Bohusz, 2014, p. 196) e, paragonando Padova a Venezia, Kazimierz Kognowicki (Kognowicki, 1783, pp. 66-67).

Nella visione di Moszyński, la decadenza riguarda gli antichi punti di forza della città: il Santo e l'Ateneo. Al secondo è dedicata l'ultima parte del brano che comincia, anche quella, con una frase che mette a confronto il passato e il presente per evidenziare una regressione: "Bisognava visitare l'università, un tempo così famosa e ora così insignificante" (ibid., p. 563; Ms., p. 59) ${ }^{5}$. In questo modo Moszyński mette in discussione la validità della tradizionale denominazione Patavium doctum, "Padova la dotta" (cf. Kowalczyk, 2005, p. 186), mentre secondo Kognowicki (1783, p. 69) questo titolo rimane valido.

Moszyński valuta senza gentilezza il corpo docente dell'università padovana, visto in blocco: "I professori sono per lo più sacerdoti di sessant'anni, il che mi è bastato" (ibid., p. 563; Ms., p. 57). Tuttavia, conobbe il colto direttore del Gabinetto di Fisica (il signor Stratico ${ }^{6}$ ) e sentì parlare del dotto capo dell'Osservatorio (padre Toaldo). Il conte menziona anche uno scienziato padovano che aveva costruito due anni prima un termometro "secondo i principi che [Moszyński considerava] di propria invenzione" (ibid., p. 563; Ms., p. 58). Il viaggiatore esprime però la convinzione che il suo strumento, costruito a Varsavia cinque anni prima (ma non reso pubblico), è migliore e più comodo, cogliendo anche l'occasione per fare una riflessione sulla precedenza e sul plagio nel campo delle invenzioni.

Moszyński critica anche altri aspetti della città. Ad esempio, secondo lui, la Sala della Ragione è molto brutta, piena di brutti affreschi e illuminata male. Questo massone e spirito illuminista dubita dell'erudizione dei monaci italiani e tinge di ironia il suo resoconto della visita alla tesoreria della basilica di Sant'Antonio, dove gli sono state mostrate diverse reliquie: "Bisognava inginocchiarsi, recitare preghiere", "Da quando viaggio, non ho mai visto niente di simile", "Non ho mai avuto una festa come questa, neanche a Loreto" (Moszyński, 1970,

5 La crisi dell'università padovana era iniziata nel XVII secolo, mentre nel XVIII secolo furono prese alcune misure per porvi rimedio (cf. Ottocentenario).

6 Simone Filippo Stratico (1733-1824), matematico, fisico e ingegnere di grande prestigio, rettore dell'Ateneo di Padova (cf. Treccani/Stratico). 
p. 561; Ms., p. 54). Come osservato da Adam Kucharski (2008, p. 171), Moszyński - accanto a Stanisław Staszic o Jan Potocki - rappresentava una tendenza ancora minoritaria, ma lentamente in crescita nella seconda metà del XVIII secolo: quella del razionalismo illuminista, che era una manifestazione della secolarizzazione della mentalità. Bisogna però notare che nella traduzione polacca del diario manca l'intero frammento, meno ironico e più informativo, che Moszyński ha dedicato al Santo:

la principale reliquia di Padova è la lingua vermiglia di Sant'Antonio, rimasta tale benché il resto del suo corpo fosse diventato polvere. [...] Si vedono ancora alcuni frammenti degli scritti di Sant'Antonio, che aveva un carattere piuttosto bello, e il suo ritratto fatto alla sua morte. Morì di idropisia all'età di 36 anni, dopo aver riportato in vita alcuni morti. Chi non conosce i miracoli deve solo fare il giro della tomba e li vedrà tutti in bassorilievi di marmo piuttosto belli. (Ms., pp. 54-55)

Il quadro del declino di Padova abbozzato da Moszyński nella tarda primavera del 1786 è in sintonia con la presentazione di questa città pubblicata nel Pamiętnik Historyczno-Polityczny del marzo 1787. Per undici mesi di quell'anno, da gennaio a novembre, questo mensile pubblicò a puntate il ciclo "Notizie sullo stato attuale dei paesi italiani". Padova, menzionata tra le altre importanti città della Repubblica di Venezia, vi è descritta come "una città famosa per diversi secoli per la sua potenza e le sue scienze, che ora presenta al viaggiatore uno spettacolo molto triste". "Deliziosi chiese e palazzi" sono solo "ricordi della prosperità passata" circondati da campi vuoti e "le strade sono strette, tortuose e fangose". Gli abitanti sono poveri e mal vestiti, mentre il suono incessante delle campane dei numerosi monasteri rende la vita insopportabile (Świtkowski, 1787, pp. 210-211).

Quattro anni dopo il soggiorno di Moszyński, il 13 giugno 1790, arrivò a Padova un altro illuminista polacco, Stanisław Staszic, che visitava l'Italia come precettore dei figli di Andrzej Zamoyski. Nel suo diario questo viaggiatore si mostra molto spesso severo verso l'Italia e nei confronti degli italiani, criticando città, costruzioni, arte, costumi, cucina, pigrizia, povertà (Wrześniak, 2010, p. 132). Per quanto riguarda Padova, come Moszyński, Staszic nota la divergenza tra le grandi 
dimensioni della città e la scarsa popolazione. Rileva anche segni del declino dell'università: mancanza di fondi per un gabinetto di storia naturale e lo stato trascurato dell'osservatorio. C'è pure una somiglianza tra la derisione di Moszyński di fronte ad alcune reliquie e le parole glupstwa (cose insensate), przesady (superstizioni) e fanatyzm (fanatismo) usate da Staszic nel commentare la processione di Sant'Antonio (Lenart, 2005, pp. 38-39; Leśniewski, 1931, pp. 47-48).

La visione dell'Italia, spesso critica, presentata su Pamiętnik Historyczno-Polityczny, provocò la reazione dell'autore del testo il cui titolo polacco può essere reso come Precauzioni per chi legge scritti storici e politici, quali memorie, diari, viaggi e simili (Piramowicz, 1787). L'anonimo, identificato come Grzegorz Piramowicz, adottando tono polemico, presentò l'Italia come "un centro di ricca vita scientifica e la patria dei più eminenti studiosi d'Europa" (Partyka, 2017, p. 162). Contrariamente a Piotr Świtkowski, editore e redattore del Pamiętnik, che non conosceva l'Italia personalmente, Piramowicz aveva visitato diversi centri accademici italiani su incarico della Commissione dell'Educazione Nazionale. Nella sua appassionata difesa dello stato delle scienze nel Bel Paese, elencò molti studiosi che rappresentavano anche la Repubblica di Venezia, compresa l'Accademia di Padova, e tra questi Giuseppe Toaldo (1719-1797), il famoso astronomo e meteorologo menzionato da Moszyński (Piramowicz, 1787, p. 43).

Małgorzata Wrześniak nota la similitudine tra l'opinione critica sugli italiani di Staszic e la descrizione del viaggio in Italia pubblicata nel 1785 dallo storico prussiano Archenholz (Wrześniak, 2010, pp. 136-137). È proprio quest'ultima ad essere evidentemente stata fonte diretta dell'immagine negativa dell'Italia presentata su Pamiętnik: la denuncia dei vizi degli italiani, quali oscurità, odio nazionale, mancanza di patriottismo, codardia, mancanza di socievolezza e ospitalità, che ha talmente indignato Piramowicz, costituisce in realtà la prima versione polacca del testo pubblicato col nome dell'autore solo nell'Ottocento (Archenholz, 1828) ${ }^{7}$. È possibile che Moszyński abbia preso da Archen-

${ }^{7}$ È la conclusione del confronto della traduzione pubblicata nel 1828 con il testo di Pamiętnik. 
holz (consultando l'originale) l'idea della decadenza della città, che per vari secoli è stata famosa per la sua potenza e la sua scienza e adesso invece presenta agli occhi del viaggiatore un'immagine molto triste:

Padwa, wsławiona kilkowieczną niepodległością i potęgą, niegdyś główne siedlisko uczoności; wystawia dziś podróżnemu smutny zmienności obraz. (Archenholz, 1828, p. 49)

Padwa Miasto, które przez kilka wieków dla swej potęgi i nauk, było sławne, wystawia teraz z siebie podróżnemu, bardzo smutny widok. (Pamiętnik, 1787 , p. 210)

Il resto della nota comporta però le impressioni e riflessioni del conte stesso.

\section{LO SPAZIO URBANO, L'ARCHITETTURA E LE BELLE ARTI}

Alla frase di apertura, che esprime l'idea della decadenza della città legata alla perdita dell'importanza del Santo e dell'Ateneo, segue la parte più positiva del brano, dedicata al Prato della Valle, che è un esempio dell'interesse del viaggiatore per la trasformazione degli spazi urbani (cf. Wyrzykowska, 2012, p. 21). Moszyński racconta di aver visitato la famosa piazza nell'ottima compagnia del promotore del progetto di rinnovamento, che cerca di ripristinare l'importanza della città, avendo cura di introdurre cambiamenti piacevoli e utili. Moszyński lo chiama "le procurateur Meno" e dice di averlo conosciuto a Roma, dove è ambasciatore, mentre prima è stato podestà di Padova (Moszyński, 1970, pp. 559-560; Ms., p. 51). Si tratta dell'illustre veneziano Andrea Memmo (1729-1793), impegnato al servizio della Serenissima (provveditore di Padova negli anni 1775-1776, ambasciatore presso la Santa Sede negli anni 1783-1786, eletto procuratore di San Marco nel 1785), architetto come Moszyński (Treccani/Memmo). È una delle quattro persone menzionate nel brano che il viaggiatore descrive come colte. Visto che Memmo gli ha mostrato due delle statue di famosi personaggi legati a Padova, disposte lungo il canale circolare sulla rinnovata piazza, quelle di Jan Sobieski ${ }^{8}$ e Jan Zamoyski, Moszyński informa Stanislao Augusto, che ha

\footnotetext{
8 Nel settembre del 1785, Katarzyna Platerowa ha individuato questa statua durante la sua visita al Prato della Valle (Platerowa, 2013, p. 97).
} 
finanziato queste statue, del loro stato di completamento ed emette un giudizio estetico favorevole? .

Tra gli edifici che Moszyński visitò a Padova il posto di rilievo viene nel suo diario dedicato alla chiesa di Santa Giustina, descritta come "la più bella e la più grande di Padova" (Moszyński, 1970, p. 560; Ms., p. 53). È un altro punto che accomuna Moszyński e Staszic. All'interno, l'attenzione del conte è concentrata su un dipinto del pittore rinascimentale Paolo Veronese (nato nel 1528 a Verona, morto nel 1588 a Venezia) raffigurante il martirio di Santa Giustina ${ }^{10}$. Il pittore è caratterizzato come eccellente e le teste delle figure descritte come "belle e vere" (ibid., p. 561; Ms., p. 53). Una valutazione simile è contenuta nel frammento dedicato a questo pittore nella relazione del soggiorno a Venezia. Moszyński, a cui "Veronese piace tanto", ha ammirato le Nozze di Cana nella chiesa di San Giorgio Maggiore, apprezzando "le teste così naturali che è impossibile non scoprire in esse la verità così spesso sacrificata alla squisitezza della forma e della pennellata o alla foga dell'immaginazione" e insistendo sul fatto che era soprattutto "la verità delle teste" a non permettergli di staccare lo sguardo dal dipinto (ibid., p. 581; Ms., p. 92).

Nel brano padovano Moszyński cita due artisti, ambedue veneti e rinascimentali. L'altro è Palladio (nato a Padova nel 1508 come Andrea di Pietro della Gondola, morto nel 1580 a Maser), che viene menzionato persino due volte. Infatti, il viaggiatore notò l'arte palladiana nella costruzione dei chiostri del monastero benedettino e nell'architettura del cortile dell'università ${ }^{11}$. L'interesse per l'arte di Palladio accomuna Moszyński ad altri viaggiatori polacchi in Italia della seconda metà del Settecento (cf. Wrześniak, 2010, p. 134). Invece Moszyński-architetto praticante il barocco (cf. Mańkowski, 1930) e Moszyński-turista appassionato di questo stile (cf. Wyrzykowska, 2012), non ha trovato a Padova oggetti corrispondenti a questo suo gusto estetico.

9 Vedi nei lavori di Mirosław Lenart (2005, 2005a) interessanti riflessioni a proposito di queste statue di illustri polacchi, in particolare congetture sui personaggi che esse rappresentano.

10 Morawska (2002, p. 134) attribuisce il quadro a Tiziano.

11 È un'attribuzione menzionata da Lalande (1769, p. 284). 


\section{IL TEATRO}

Il teatro era una delle passioni di Moszyński, che fu incaricato da Stanislao Augusto di supervisionare il Teatro Nazionale di Varsavia (fondato nel 1765) agli inizi della sua attività. Dopo la riapertura del teatro nel 1774, Moszyński ricoprì la posizione di sovraintendente, poi agì come intermediario a nome del re nei negoziati riguardanti, tra l'altro, le sovvenzioni per il teatro. Presentò al re diversi memorandum sul funzionamento del teatro e negli anni Ottanta si occupava dell'Associazione degli Attori Polacchi. Finanziò alcuni balletti, decorazioni e costumi, probabilmente scrisse anche un'opera teatrale. Fu sotto i suoi auspici che Wojciech Bogusławski iniziò la sua attività di attore e regista (Wierzbicka-Michalska, 1977).

Non è dunque sorprendente che il diario di Moszyński riporti diverse osservazioni sulla vita teatrale italiana. "In ogni città italiana c'è almeno un teatro, a volte ce ne sono due o anche tre" (Moszyński, 1970, p. 273). A Firenze "due e spesso quattro teatri danno spettacoli svariati" (ibid., p. 556). "I romani adorano gli spettacoli, ma gli spettacoli a Roma possono aver luogo solo durante il Carnevale, quindi ogni celebrazione in chiesa diventa uno spettacolo." (ibid., p. 455). A Napoli, "una rappresentazione di ogni nuova opera è riservata esclusivamente [ai monaci]" (ibid., pp. 463-464). A Venezia, famosa per il gran numero di teatri e l'intensità della vita teatrale, Moszyński soggiorna in un periodo in cui non può goderne: "Per l'Ascensione c'è solo un teatro aperto, inoltre di solito è odioso: ci si va solo per vedere il balletto" (ibid., p. 567; Ms., p. 66). Per quanto riguarda il teatro visitato da Moszyński a Padova, le informazioni riportate nel diario riguardano solo le sue dimensioni: "Il teatro che ho visto è grande; ha cinque file di palchi, trentadue per piano" (ibid., p. 562; Ms., p. 56). Si tratta sicuramente del Teatro Nuovo, costruito a metà del XVIII secolo ${ }^{12}$. C'erano infatti cinque file di palchi, però 29 in ogni fila (Brunelli, 1921, pp. 151, 155) e non 32, come notato da Moszyński, forse a causa della sua scarsa vista.

12 Teatro Nuovo e della Nobiltà (oggi Il Teatro Verdi). Cf. Teatro Verdi. 
Moszyński non dà alcuna informazione sullo spettacolo, perché non ce n'erano durante la sua prima visita a Padova. Non è invece escluso che durante il secondo soggiorno abbia visto uno dei due messi in scena durante la Fiera del giugno del 1786: Medonte re d'Epiro o Zamira ${ }^{13}$.

Nella parte del diario dedicata a Venezia, Moszyński approfondisce le circostanze della villeggiatura, il suo legame con la vita teatrale del Veneto e, su questo sfondo, l'importanza del teatro di Padova:

Il grande caldo spopola Venezia di coloro che possono andarsene. Anche prima della fine della fiera dell'Ascensione, che dura quindici giorni, la maggior parte della gente va in campagna. Gli spettacoli cessano per quattro mesi. La città fiorisce di nuovo solo alla fine di ottobre [...]. L'aria diventa densa, i canali puzzano; dopo la festa del Corpus Domini, non c'è più nessuno spettacolo, nessuna celebrazione con processione. Quindi, per forza di cose, bisogna scambiare la città con la campagna, cioè andare nelle prossimità delle piccole città della terraferma, da dove si va a teatro a Padova, a Mestre, a Treviso. Tuttavia nessun nobile veneziano può, senza il permesso del senato, andare a Bologna, Modena, Milano e altre città dove ci sono teatri, mentre i nobili della terraferma vanno dove vogliono. [...] Torniamo alla nostra villeggiatura. Questo è il momento che attira tutto il beau monde sulle rive del Brenta per essere più vicino a Padova (pp. 597-599; Ms., pp. 123-126).

Brevi informazioni sull'edificio e sulla sala del teatro padovano hanno dato luogo a una riflessione comparativa sulla vita teatrale in Italia e in Polonia. Per quanto riguarda l'Italia, Moszyński critica la mania di costruire grandi teatri, che deriva dal fatto che "ogni famiglia un po' illustre vuole avere il suo palco permanente" (Moszyński, 1979, p. 562; Ms., p. 56). Le circostanze della creazione del Teatro Nuovo di Padova sono una buona illustrazione di questa osservazione. Questo teatro, chiamato Teatro Nuovo e della Nobiltà, fu fondato proprio su iniziativa e grazie agli sforzi di un'associazione di rappresentanti della nobiltà locale.

13 Il primo rappresentato dal 12 giugno, il secondo dal 20 giugno (Brunelli, 1921, p. 197). Ivi anche l'informazione che al di fuori del carnevale a Padova gli spettacoli teatrali erano allestiti solo durante la fiera. 
Moszyński confronta la vita teatrale italiana e polacca in termini di spaccato sociale del pubblico (più ampio in Italia) e della frequenza delle prime teatrali (maggiore in Polonia). Egli nota che mettere in scena una nuova opera ogni mese o ogni sei settimane è rovinoso per l'imprenditore teatrale e stancante per gli attori. Questa riflessione è motivata dalla notizia "sui cambiamenti nella gestione del nostro teatro di Varsavia" (ibid., p. 562; Ms., p. 57) ricevuta dal viaggiatore. Di cosa potrebbe trattarsi in queste osservazioni rivolte al re? Ebbene, a cavallo tra il 1785 e il 1786 Wojciech Bogusławski firmò un contratto con Franciszek Ryx perché la sua compagnia si esibisse a Varsavia da maggio, ma verso il 15 maggio "il contratto tra Ryx e Bogusławski fu rotto. Il rappresentante di Ryx tolse a Bogusławski gli attori sotto contratto" (Kott, 1967, p. 58)

\section{EVENTI}

Durante il primo soggiorno a Padova Moszyński ebbe l'opportunità di ascoltare e osservare delle esercitazioni militari. Commenta questo spettacolo ironicamente, deridendo la voce e i gesti del comandante. Alla fine del diario, Moszyński fa il resoconto di uno spettacolo più complesso, la corsa dei barberi sul Prato della Valle, menzionata già nel primo brano ${ }^{14}$. Questo evento è l'unico elemento riportato dal viaggiatore della sua seconda visita a Padova, connessa al fatto che, facendo come gli abitanti di Venezia all'inizio dell'estate, Moszyński è andato in villeggiatura sul Brenta, "per essere più vicino a Padova". Anche al Prato della Valle Moszyński segue gli altri, cioè fa "venti volte" il giro della piazza prima di prender posto in una finestra dalla quale può seguire la corsa grazie a un cannocchiale acromatico. Nell'ultima frase del diario, parla dell'orgoglio che i padovani hanno del loro palio, in cui, grazie alla forma ellittica dell'immensa piazza, la corsa dei cavalli è visibile tutto il tempo, e non solo un attimo, come altrove. Moszyński non abbandona

14 Le corse di cavalli a Padova vengono menzionate da Morawska (2002, p. 215), fermatasi a Padova per una notte proprio per visitare la città al tempo della Fiera del Santo che attirava moltissimi veneti e stranieri. 
però il suo atteggiamento critico e malizioso, quando nota le brutte case che circondano la bella piazza, i vecchi tappeti che ornano i balconi molto stretti, i cabriolet molto brutti, e quando riconosce la truppa di soldati derisa qualche settimana prima, per via del comandante che dava ordini come se stesse facendo una predica.

Le due visite al Prato della Valle sono un esempio di quanto il conte amasse scoprire gli stessi luoghi varie volte, da vari punti di vista, nonché della vivacità, della perspicacia e del carattere soggettivo della sua relazione (cf. Kostkiewiczowa, 1988, pp. 208-210).

\section{FINE DEL VIAGGIO}

L'ultima voce del suo diario riguarda Padova, ma sia il viaggio italiano che le peregrinazioni terrene del conte Moszyński ebbero fine a Venezia.

In vari studi a lui dedicati si riporta che morì a Padova o Venezia, nel giugno o luglio $1786^{15}$. L'esatta data (il 3 luglio) e il luogo corretto (Venezia) della morte del viaggiatore vengono riportati da Piotr Biliński, in base alla lettera di Ludwik Hempel a Gaetano Ghigiotti scritta da Venezia 1'8 agosto 1786, conservata (collocazione: 479a) nell'Archivio Ghigiotti dell'AGAD ovvero l'Archivio Centrale dei Documenti Storici di Varsavia (Biliński, 2006, p. 47). Altri documenti che fanno parte dello stesso archivio (collocazione: $496 \mathrm{a})^{16}$ permettono di confermare questi dati fondamentali e forniscono ulteriori dettagli sulle circostanze della morte di Moszyński. Si tratta delle lettere scritte il 5 e il 12 luglio sempre a Ghigiotti, ma questa volta da Marianna, la moglie di Giuseppe Dall'Oglio, agente del re di Polonia a Venezia, nelle quali si legge che il conte morì nella rinomata locanda del Leon Bianco, in seguito a un violento attacco di colica biliare. Più ampiamente tratterò questo argomento in un'altra sede.

15 "Morì probabilmente a Padova, nella seconda metà di giugno o all'inizio del luglio 1786." (Wierzbicka-Michalska, 1977, p. 111; IPSB/Moszyński); "a Padova” (Wyrzykowska, 2012, p. 20), "l’11 giugno 1786 a Venezia” (Mańkowski, 1930, p. 227; AM).

${ }^{16}$ Ringrazio Małgorzata Ewa Kowalczyk per avermeli segnalati. 


\section{CONCLUSIONI}

Come sottolineato da Krzysztof Pomian (Calafat, 2010, p. 12), gli interessi di Moszyński erano veramente enciclopedici. L'analisi dei brani del diario relativi ai soggiorni a Padova conferma la versatilità del viaggiatore che scrive da esperto di architettura e di spazi urbani, conoscitore di teatro, costruttore di strumenti scientifici, ammiratore di artisti rinascimentali, amante della verità nell'arte, osservatore acuto, critico e ironico della vita culturale e scientifica. Nell'immagine tracciata dalla sua penna, dove elementi descrittivi si intrecciano con impressioni estetiche, valutazioni e digressioni, Padova risulta una città decaduta ma non priva di personaggi colti e strettamente legata a Venezia non solo politicamente, ma anche dall'itinerario di Moszyński. Sulla scia di altri relazioni di viaggio, il conte descrive Padova come ampia ma poco popolata, ma poi il viaggiatore si diletta a inserire nella sua relazione elementi di reportage, cogliendo la vita padovana sul vivo. La sua relazione illustra bene l'approccio critico e razionale dell'autore, la sua devozione al re di Polonia, il suo forte legame con il teatro di Varsavia. Tre volte il pensiero del viaggiatore va a persone, ambienti e attività di cui si è occupato prima del viaggio, il che contribuisce a dare al testo un taglio personale. La scelta di concentrarsi sulle proprie impressioni piuttosto che sui monumenti, insieme alla brevità del tempo dedicato a Padova e alla vista debole del viaggiatore, possono spiegare il fatto che Moszyński non fa nessuna menzione di alcuni luoghi padovani molto famosi quali l'orto botanico o la cappella degli Scrovegni, ma non li menzionano neppure Franciszek Ksawery Bohusz e Katarzyna Platerowa che, come Moszyński, visitarono Padova negli anni Ottanta del Settecento.

\section{BIBLIOGRAFIA}

Archenholz, J.W. (1828). Wtochy przez Archenholca w dwóch częściach ttumaczone z niemieckiego. Płock: Nakładem i drukiem Karola Kuliga. Barycz, H. (1965). Spojrzenia w przeszłość polsko-wtoska. Wrocław: Zakład Narodowy im. Ossolińskich. 
Biliński, P. (2006). Moszyńscy: studium z dziejów łoniowskiej linii rodu w XIX wieku. Kraków: Uniwersytet Jagielloński - Biblioteka Jagiellońska.

Bohusz, F.K. (2014). Dzienniki podróży (edited by F. Wolański). KrakówWrocław: Księgarnia Akademicka.

Brunelli, B. (1921). I teatri di Padova dalle origini alla fine del secolo XIX. Padova: Libreria Angelo Draghi.

Calafat, G. (Ed.). (2010). August Fryderyk Moszyński (1731-1786). Journal de voyage. I. La France (1784-1785). Paris: CNRS/Alain Bourdy et $\mathrm{C}^{\mathrm{ie}}$.

Kognowicki, K. (1783). Droga rzymska z nawrotem do swojej ojczyzny nie bez gościńca nazad powróconego. Warszawa: Drukarnia J.K.M. u S. Krzyża.

Kostkiewiczowa, T. (1988). L'Italie vue par des Polonais éclairés. Relation des voyages de Stanisław Kostka Potocki et d'August Moszyński. In E. Kanceff, \& R. Lewanski (Eds.), Viaggiatori polacchi in Italia (pp. 203-216). Genève: Slatkine.

Kott, J. (Ed.). (1967). Teatr Narodowy 1765-1794. Warszawa: PIW.

Kowalczyk, M.E. (2005). Obraz Włoch w polskim piśmiennictwie geograficznym i podróżniczym osiemnastego wieku. Toruń: Adam Marszałek.

Kucharski, A. (2008). Czy wszyscy staropolscy peregrynanci wierzyli w cuda i relikwie? Uwagi na marginesie dzienników podróży XVII i XVIII wieku. In J. Wiśniewski (Ed.), Laicyzacja i sekularyzacja społeczeństwa nowożytnego (XVI-XVIII w.) (pp. 155-171). Olsztyn: „Hosianum”.

Lalande, J. de (1769). Voyage d'un français en Italie, fait dans les années 1765 et 1766, t. 8. Paris: Desaint. Retrieved from https://gallica.bnf.fr.

Lenart, M. (2005). Polscy podróżnicy w padewskiej bazylice św. Justyny $(X V I-X X$ w.). Opole.

Lenart, M. (2005a). Il mistero della statua in Prato della Valle, Padova e il suo territorio, $X X, 118$, novembre-dicembre, 13-16.

Leśniewski, C. (Ed.). (1931). Dziennik podróży Stanisława Staszica 1789-1805. Kraków: Księgarnia Gebethnera i Wolffa.

Mańkowski, T. (1930). August Moszyński, architekt polski XVIII stulecia. In Prace Komisji Historii Sztuki, t. 4 (pp. 169-230). Kraków: Polska Akademia Umiejętności.

Morawska, T.K. z Radziwiłłów (2002). Diariusz podróży europejskiej w latach 1773-1774 (edited by B. Rok). Wrocław: Wydawnictwo Uniwersytetu Wrocławskiego. 
Moszyński, A. (1930). Voyage en Provence d'un gentilhomme polonais, 1784 -1785, Le comte Moszynski (edited by F. Benoit). Marseille: Institut historique de Provence.

Moszyński A.F. (1970). Dziennik podróży do Francji i Włoch 1784-1786 (B. Zboińska- Daszyńska, Trans.). Kraków: Wydawnictwo Literackie. [Świtkowski, P.] (1787). Dokończenie wiadomości o Wenecyi, Pamiętnik Historyczno-Polityczny, VI, 3 (marzo), 193-214.

Partyka, M. (2017). Przestrogi dla Pamiętnika. O dwóch wizjach Włoch ma łamach publicystyki oświeceniowej. In L. Masi, E. NicewiczStaszowska, J. Pietrzak-Thébault, \& M. Woźniewska-Działak (Eds.), Polska $i$ Włochy $w$ dialogu kultur / La Polonia e l'Italia nel dialogo tra le culture (pp. 155-168). Warszawa: Wydawnictwo Naukowe UKSW.

Platerowa, K. z Sosnowskich. (2013). Moja podróż do Włoch. Dziennik z lat 1785-1786 (edited by M.E. Kowalczyk). Łomianki: LTW.

[Piramowicz, G.] (1787). Przestrogi dla czytajacych pisma historyczno-polityczne, jako to pamiętniki, dzienniki, wojaże, geografie i tym podobne. Warszawa.

Pomian, K. (2010). Préface. In G. Calafat (Ed.), August Fryderyk Moszyński (1731-1786). Journal de voyage. I. La France (1784-1785). Paris: CNRS/Alain Bourdy et $\mathrm{C}^{\mathrm{ie}}$.

Rok, B., \& Wolański, F. (Eds.). (2016). Staropolskie podróżowanie. Kraków: Księgarnia Akademicka.

Wierzbicka-Michalska, K. (1977). Moszyński August Fryderyk (dictionary entry). In E. Rostworowski (Ed.), Polski słownik biograficzny, t. 22 (pp. 108-112). Wrocław-Warszawa-Kraków-Gdańsk: Zakład Narodowy im. Ossolińskich.

Wrześniak, M. (2010). Podróż włoska Stanisława Staszica: kilka słów o guście estetycznym polskiego peregrynanta u schyłku osiemnastego wieku. Saeculum christianum, 17(2), 129-164.

Wyrzykowska M. (2012). Percepcja barokowych dzieł sztuki w XVIII wieku w świetle Dziennika podróży do Francji i Włoch w latach 1784-1786 Augusta Moszyńskiego. Quart, 1, 19-43.

AGAD/Ghigiotti: http://www.agad.gov.pl/pomoce/AGhigiott15.xml AM: http://www.encyklopediateatru.pl/osoby/79127/august-moszynski

IPSB/Moszyński: https://www.ipsb.nina.gov.pl/a/biografia/august-fryderykmoszynski-h-nalecz 
Ottocentenario: https://www.ottocentenariouniversitadipadova.it/timeline/ilsettecento/

Teatro Verdi: http://www.teatrostabileveneto.it/sedi/teatro-verdi/

Treccani/Memmo: http://www.treccani.it/enciclopedia/andrea-memmo_(Dizionario-Biografico)

Treccani/Stratico: https://www.treccani.it/enciclopedia/simone-filippo-stratico \%28Dizionario-Biografico\%29/Wiki/1786: https://pl.wikipedia.org/ wiki/1786

Riassunto: L'articolo è dedicato all'immagine di Padova nel Diario di viaggio in Francia e in Italia (1784-1786) di August Fryderyk Moszyński (1731-1786), stretto collaboratore del re Stanislao Augusto Poniatowski, architetto, direttore di edifici reali, promotore del teatro di Varsavia. Il diario è stato parzialmente pubblicato in francese $(1930,2010)$ e in traduzione polacca (1970). Nella letteratura odeporica polacca del Settecento si distingue per il suo approccio critico, il carattere quasi scientifico e l'interesse per il presente. Una volta fissate le date dei soggiorni a Padova, vengono analizzati elementi quali descrizioni, impressioni e valutazioni relative ad ambienti urbani, edifici, quadri, persone ed eventi, nonché la vita culturale e scientifica. Il ritratto di Padova disegnato da Moszyński è messo a confronto con altri odeporici dell'epoca e quello che emerge da testi su argomenti simili presentati al pubblico polacco nello stesso periodo, in particolare con la descrizione dell'Italia apparsa a puntate sulla rivista varsaviana Pamiętnik Historyczno-Polityczny nel 1787. L'analisi dei brani del diario relativi ai due soggiorni a Padova conferma la versatilità del viaggiatore. La descrizione di Padova è sfaccettata, spesso ironica e personale. Moszyński scrive da esperto di architettura e di spazi urbani, conoscitore di teatro, costruttore di strumenti scientifici, ammiratore di artisti rinascimentali, amante della verità nell'arte, osservatore acuto e severo. L'ultima parte del saggio è dedicata alle circostanze della morte di Moszyński, che - contrariamente a quanto affermato in alcune fonti e a quanto suggerito dal diario stesso - non è avvenuta a Padova, bensì a Venezia (il 3 luglio 1786, nella famosa locanda del Leon Bianco).

Parole chiave: August Fryderyk Moszyński, Padova, diario di viaggio, Italia, immagine letteraria 